\title{
Cost analysis of non-invasive fractional flow reserve derived from coronary computed tomographic angiography in Japan
}

\author{
Takeshi Kimura $\cdot$ Hiroki Shiomi $\cdot$ Sachio Kuribayashi $\cdot$ Takaaki Isshiki • \\ Susumu Kanazawa $\cdot$ Hiroshi Ito $\cdot$ Shunya Ikeda $\cdot$ Ben Forrest $\cdot$ Christopher K. Zarins • \\ Mark A. Hlatky $\cdot$ Bjarne L. Norgaard
}

Received: 12 May 2014 / Accepted: 26 June 2014/Published online: 17 July 2014

(C) Japanese Association of Cardiovascular Intervention and Therapeutics 2014

\begin{abstract}
Percutaneous coronary intervention (PCI) based on fractional flow reserve $\left(\mathrm{FFR}_{\text {cath }}\right)$ measurement during invasive coronary angiography (CAG) results in improved patient outcome and reduced healthcare costs. FFR can now be computed non-invasively from standard coronary CT angiography (cCTA) scans $\left(\mathrm{FFR}_{\mathrm{CT}}\right)$. The purpose of this study is to determine the potential impact of non-invasive $\mathrm{FFR}_{\mathrm{CT}}$ on costs and clinical outcomes of patients with suspected coronary artery disease in Japan. Clinical data from 254 patients in the HeartFlowNXT trial, costs of goods and services in Japan, and clinical outcome data from the literature were used to estimate the costs and outcomes of 4 clinical pathways: (1) CAG-visual guided

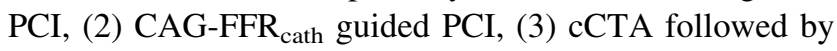
CAG-visual guided PCI, (4) cCTA-FFR ${ }_{\mathrm{CT}}$ guided PCI. The CAG-visual strategy demonstrated the highest projected
\end{abstract}

T. Kimura $(\bowtie) \cdot$ H. Shiomi

Department of Cardiovascular Medicine, Kyoto University

Graduate School of Medicine, 54 Shogoin Kawahara-cho,

Sakyo-ku, Kyoto 606-8507, Japan

e-mail: taketaka@kuhp.kyoto-u.ac.jp

S. Kuribayashi

Department of Radiology, Keio University School of Medicine,

Tokyo, Japan

T. Isshiki

Department of Cardiology, Teikyo University Hospital, Tokyo, Japan

S. Kanazawa

Departments of Radiology, Okayama University Graduate

School of Medicine, Okayama, Japan

H. Ito

Departments of Cardiology, Okayama University Graduate

School of Medicine, Okayama, Japan cost $(\$ 10,360)$ and highest projected 1-year death/myocardial infarction rate $(2.4 \%)$. An assumed price for $\mathrm{FFR}_{\mathrm{CT}}$ of US $\$ 2,000$ produced equivalent clinical outcomes (death/MI rate: $1.9 \%)$ and healthcare costs $(\$ 7,222)$ for the cCTA-FFR $\mathrm{CT}_{\mathrm{T}}$ strategy and the CAG-FFR ${ }_{\text {cath }}$ guided PCI strategy. Use of the cCTA-FFR ${ }_{\mathrm{CT}}$ strategy to select patients for PCI would result in $32 \%$ lower costs and $19 \%$ fewer cardiac events at 1 year compared to the most commonly used CAG-visual strategy. Use of cCTA-FFR ${ }_{\mathrm{CT}}$ to select patients for CAG and PCI may reduce costs and improve clinical outcome in patients with suspected coronary artery disease in Japan.

Keywords Fractional flow reserve - Non-invasive diagnosis · Cost-effectiveness · Computational fluid dynamics · Coronary computed tomographic angiography

\author{
S. Ikeda \\ International University of Health and Welfare, Tokyo, Japan \\ B. Forrest · C. K. Zarins \\ HeartFlow, Inc., Redwood City, CA, USA
}

M. A. Hlatky

Department of Health Research and Policy, Stanford University School of Medicine, Stanford, CA, USA

B. L. Norgaard

Department of Cardiology, Aarhus University Hospital Skejby, Aarhus, Denmark 


\section{Introduction}

Prior studies have shown clinical and economic benefits from assessing and utilizing invasive fractional flow reserve $\left(\mathrm{FFR}_{\mathrm{cath}}\right)$ measurements to guide percutaneous coronary intervention (PCI). In the randomized controlled fractional flow reserve versus angiography for multivessel evaluation (FAME) study including 1,005 patients, it was demonstrated that deferring PCI in vessels not associated with myocardial ischemia based on $\mathrm{FFR}_{\text {cath }}$ resulted in improved clinical outcomes and lower costs [1, 2]. Furthermore, the FAME II study involving 888 patients demonstrated that PCI in vessels associated with myocardial ischemia based on FFR $_{\text {cath }}$ significantly reduced urgent revascularization when compared to medical therapy alone [3]. In all studies published to date, invasive FFR has been assessed during angiography (FFR $\left.\mathrm{Fath}_{\text {ch }}\right)$. While $\mathrm{FFR}_{\text {cath }}$ is widely recommended and offers clinical and economic benefits, it is not yet widely used due to inconvenience and costs [4].

A new technology based on standard coronary computed tomographic angiography (cCTA) allows FFR to be estimated non-invasively $\left(\mathrm{FFR}_{\mathrm{CT}}\right)$ before sending a patient to angiography. Three prospective, multicenter, validation studies have been performed (DISCOVER-FLOW-diagnosis of ischemia-causing stenosis obtained via non-invasive fractional flow reserve [5]; DeFACTO_determination of fractional flow reserve by anatomic computed tomographic angiography [6]; and HeartFlowNXT-HeartFlow analysis of coronary blood flow using CT angiography: NeXT steps [7] to determine the diagnostic accuracy of $\mathrm{FFR}_{\mathrm{CT}}$ using $\mathrm{FFR}_{\text {cath }}$ as the reference standard. Good concordance between $\mathrm{FFR}_{\mathrm{CT}}$ and $\mathrm{FFR}_{\text {cath }}$ was found with high diagnostic accuracy of $\mathrm{FFR}_{\mathrm{CT}}$ for the detection or exclusion of hemodynamically significant stenosis using FFR $\leq 0.80$ as the reference standard.

An analysis of potential costs and consequences of utilizing $\mathrm{FFR}_{\mathrm{CT}}$ to guide clinical decision-making in the United States has suggested the possibility of meaningful cost savings and clinical benefits [8]. In the present paper, we report a similar analysis, using data from the most recently published trial, HeartFlowNXT [7], as well as Japanese procedure and device cost information. The primary objective of this analysis is to determine the potential magnitude of cost savings and clinical benefit which could be expected in Japan through utilization of FFR $\mathrm{CT}_{\text {. }}$.

\section{Methods}

We used data from 254 patients enrolled in the HeartFlowNXT trial [7]. All patients had known or suspected stable coronary artery disease (CAD) and were scheduled for coronary angiography (CAG). Each patient in the trial was evaluated with cCTA, FFR ${ }_{\mathrm{CT}}$, clinically-indicated CAG and FFR ${ }_{\text {cath }}$. Calculation of $\mathrm{FFR}_{\mathrm{CT}}$ from standard acquired cCTA images and evaluation of $\mathrm{FFR}_{\text {cath }}$ were performed independently at core laboratories [7]. The study results demonstrated that $\mathrm{FFR}_{\mathrm{CT}}$ provided high per-patient diagnostic accuracy ( $81 \%$ ) for the detection of hemodynamically significant CAD with a sensitivity of $86 \%$ (95\% CI 77-92\%) and specificity of $79 \%$ (95 CI 72-84\%) using FFR $_{\text {cath }}$ as the reference standard. FFR ${ }_{\mathrm{CT}}$ also provided excellent discrimination of patients with and without lesion-specific ischemia with an area under the receiver-operating characteristics curve of $0.90(95 \% \mathrm{CI}$ $0.87-0.94 \%$ ) [7]. Using patient-specific data from this study, we modeled four hypothetical diagnostic/treatment pathways for patients with known or suspected CAD who are scheduled for coronary angiography (Fig. 1):

1. Pathway 1: CAG-visual: all patients undergo coronary angiography as scheduled. Those with $\geq 50 \%$ stenosis by visual assessment of angiographic images undergo PCI.

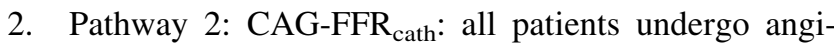
ography as scheduled. Those patients with $\geq 50 \%$ stenosis undergo $\mathrm{FFR}_{\text {cath }}$ and only those with $\mathrm{FFR}_{\text {cath }}$ $\leq 0.80$ undergo PCI.

3. Pathway 3: cCTA-CAG: all patients undergo cCTA. Only those with $\geq 50 \%$ cCTA stenosis undergo CAG. Those with $\geq 50 \%$ stenosis by visual assessment of the angiogram undergo PCI.

4. Pathway 4: cCTA-FFR ${ }_{\mathrm{CT}}-\mathrm{CAG}$ : all patients undergo cCTA. Those with $\geq 50 \%$ stenosis by cCTA undergo FFR $_{\mathrm{CT}}$. Only those with $\mathrm{FFR}_{\mathrm{CT}} \leq 0.80$ undergo $\mathrm{CAG}$ and PCI is performed after visual angiographic confirmation of the stenosis.

For each pathway, we estimated the costs in Japan in accord with the Japanese Ministry of Health, Labour and Welfare (MHLW) medical insurance medical expense database [9]. We considered cost data from the detailed economic analysis in the 2 years follow-up report of the FAME study [1] and applied the conversion rate of 93.42 Yen/Dollar. The peri-procedural costs utilized in this study are shown in Table 1. While the actual cost of FFR $_{\mathrm{CT}}$ analysis has not yet been determined, for the purposes of this analysis, we sought the cost of $\mathrm{FFR}_{\mathrm{CT}}$ which produced equivalence between the costs of Pathway 2 and Pathway 4. This was calculated to be $\$ 2,000$, which is comparable to the $\mathrm{FFR}_{\text {cath }}$ disposable costs $+\mathrm{CAG}$ procedure fee as reimbursed by the MHLW medical insurance. The analysis of each pathway involved totaling the costs for each test and procedure described for all patients in that pathway and dividing by the total number of patients (254) giving the average cost per patient. 


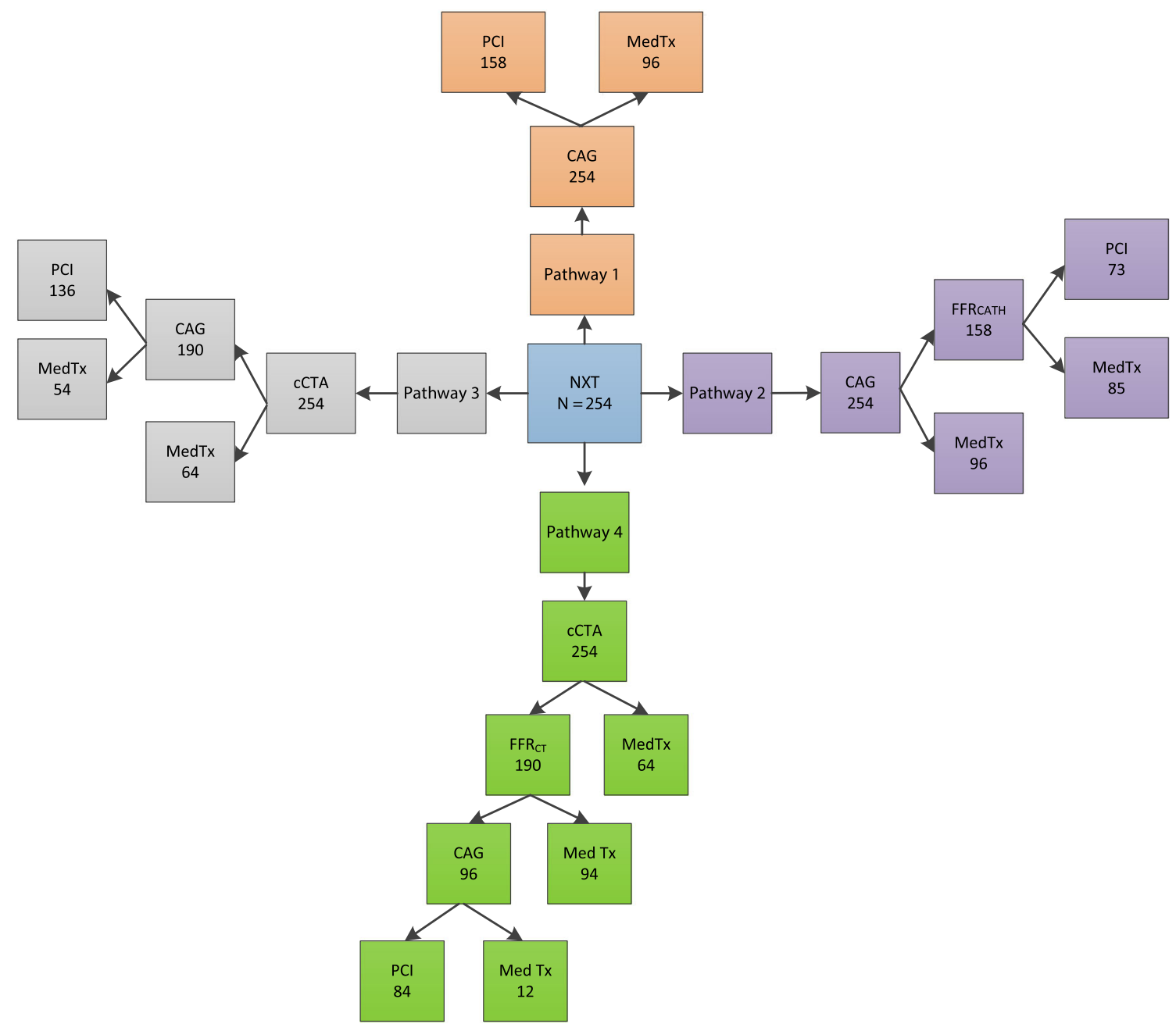

Fig. 1 Outline of 4 hypothetical diagnostic/treatment strategies applied to the 254 patient population from the HeartFlowNXT clinical trial

Table 1 Procedural costs used in the analysis

\begin{tabular}{llllllr}
\hline Costs per procedure & Procedure fee & Device cost & \multicolumn{2}{l}{ Hospital stay } & \multirow{2}{*}{ Total costs } \\
\cline { 4 - 6 } & & & & Per night & Avg nights & Total \\
\hline Angio & $\$ 420$ & $\$ 60$ & $\$ 1,500$ & 1.4 & $\$ 2,100$ & $\$ 2,580$ \\
PCI-1 vessel & $\$ 2,550$ & $\$ 5,789$ & $\$ 1,500$ & 2.0 & $\$ 3,000$ & $\$ 11,339$ \\
PCI-2 vessel & $\$ 2,550$ & $\$ 9,802$ & $\$ 1,500$ & 2.0 & $\$ 3,000$ & $\$ 15,352$ \\
PCI-3 vessel & $\$ 2,550$ & $\$ 13,815$ & $\$ 1,500$ & 2.0 & $\$ 3,000$ & $\$ 19,365$ \\
cCTA & $\$ 400$ & - & - & 0.0 & - & $\$ 400$ \\
FFR & $\$ 42$ & $\$ 1,800$ & - & 0.0 & - & $\$ 1,842$ \\
Price FFRct & - & $\$ 2,000$ & - & 0.0 & - & $\$ 2,000$ \\
\hline
\end{tabular}

We estimated future event rates for appropriately and inappropriately treated patients using the FFR cutoff value of $<0.80$ drawing on data from the deferral versus performance of PTCA in patients without documented ischemia (DEFER) [10], FAME [11, 12] and providing regional observations to study predictors of events in the coronary tree (PROSPECT) [13] studies. We considered a coronary lesion to be significant by visual assessment of the CAG or cCTA if lumen stenosis was $\geq 50 \%$ and defined functional significance of a lesion as either an $\mathrm{FFR}_{\text {cath }}$ or $\mathrm{FFR}_{\mathrm{CT}}$ of $\leq 0.80$. Accordingly, we used the following assumptions to estimate the combined 1 year death/MI rate for: (a) PCI in patients with FFR $_{\text {cath }} \leq 0.80: 3 \%$; (b) PCI in patients with FFR $_{\text {cath }}>0.80: 3 \%$; (c) medical therapy in patients with 
FFR $_{\text {cath }}>0.80: 1 \%$; (d) medical therapy in patients with FFR $_{\text {cath }} \leq 0.80: 5 \%$; (e) invasive measurement of FFR ${ }_{\text {cath }}$ : $0.4 \%[7,14]$.

\section{Results}

A total of 254 patients from the international, multicenter HeartFlowNXT trial had complete information and were included in the analysis. Fifty-seven patients (22\%) in the study were from Japanese clinical sites. Patient characteristics are shown in Table 2. One-third of the patients (80/ 254) had ischemia-causing stenoses with FFR $\leq 0.80$ and $21 \%$ of the 484 vessels in which invasive FFR was measured had FFR $_{\text {cath }} \leq 0.80$.

\section{Utilization and costs}

In this analysis, we modeled the expected cost to treat each patient. The overall cost for each patient is dependent on the test(s) utilized in a given pathway, the lesion measurements for each test, the order in which tests are performed and the treatment performed in accord with the test results. As discussed above, in this analysis we included four hypothetical clinical pathways. The average perpatient cost, for each clinical pathway is shown on Table 3 .

In the most commonly used clinical strategy, CAG visual (Pathway 1), all patients would undergo CAG, $62 \%$ would undergo PCI and 80 vessels per 100 patients would be treated based on visual assessment of the angiogram

Table 2 Characteristics of study population $(n=254)$

\begin{tabular}{ll}
\hline Age \pm SD (range) & $64 \pm 10$ years (32-84 years) \\
Men:women (\%) & $162: 92(64: 36 \%)$ \\
Asian:Caucasian (\%) & $86: 163(34: 64 \%)$ \\
Hyperlipidemia (\%) & $200(79 \%)$ \\
Hypertension (\%) & $174(69 \%)$ \\
Diabetes (\%) & $58(23 \%)$ \\
Current smoking (\%) & $46(18 \%)$ \\
Prior myocardial infarction (\%) & $5(2 \%)$ \\
Angina within past month (\%) & $197(78 \%)$ \\
\hline
\end{tabular}

with an average cost of $\$ 10,360$ per patient. In Pathway 2, $\mathrm{FFR}_{\text {cath }}$ was used to select patients for PCI and only $29 \%$ of patients would undergo PCI with 37 vessels per 100 patients requiring $\mathrm{PCI}$, assuming strict adherence to the recommended threshold of $\mathrm{FFR}_{\text {cath }} \leq 0.80$. This $54 \%$ reduction in PCI would result in a potential average cost of $\$ 7,222$ per patient corresponding to a $30 \%$ savings per patient when compared to Pathway 1. In Pathway 3, cCCTA was used to select patients for CAG reducing the number of angiograms by $25 \%$ and the number of patients undergoing PCI by $13 \%$ compared to Pathway 1 (CAG visual). PCI was guided by visual $\mathrm{CAG}$ and the number of vessels treated was reduced by only $10 \%$ with average cost savings of $12 \%$ relative to Pathway 1 . In Pathway 4, the strategy of initial cCCTA with $\mathrm{FFR}_{\mathrm{CT}}$ in patients with $\geq 50 \%$ stenosis and CAG only in those with $\mathrm{FFR}_{\mathrm{CT}} \leq 0.80$ reduced the number of angiograms by $62 \%$ and number of patients undergoing PCI by $47 \%$. Only 48 vessels per 100 patients needed PCI with an average cost per patient of $\$ 7,222$, a $30 \%$ cost savings per patient compared to Pathway 1.

\section{Clinical events}

The estimated one-year rate of death or MI for the CAG visual-guided strategy (Pathway 1) was $2.4 \%$ (Table 3). Use of cCTA to select patients for CAG (Pathway 3) reduced the death/MI rate to $2.2 \%$ due to the reduction in number of CAG. The use of FFR $\mathrm{Fath}_{\text {ar }}$ orFR $\mathrm{CT}_{\mathrm{T}}$ to guide PCI treatment (Pathways 2 and 4) resulted in the greatest reduction $(21 \%)$ in death/MI rate to $1.9 \%$ compared to visual angiography-guided treatment. The combined effects of cost and clinical event rate are shown in Fig. 2.

\section{Discussion}

Three prospective, multicenter clinical trials, comprising 609 patients and 1,050 vessels have evaluated the diagnostic accuracy of $\mathrm{FFR}_{\mathrm{CT}}$ using $\mathrm{FFR}_{\text {cath }}$ as the reference standard [5-7]. Each study has shown FFR $_{\mathrm{CT}}$ to have high diagnostic accuracy for the diagnosis of lesion-specific
Table 3 Peri-procedural costs and 1 year clinical event rates

\begin{tabular}{lllll}
\hline & $\begin{array}{l}\text { CAG } \\
\text { visual }\end{array}$ & $\begin{array}{l}\text { CAG- } \\
\text { FFR }_{\text {cath }}\end{array}$ & $\begin{array}{l}\text { cCTA- } \\
\text { CAG }\end{array}$ & $\begin{array}{l}\text { cCTA-FFR } \\
\text { CAG }\end{array}$ \\
\hline $\begin{array}{l}\text { No. of patients undergoing CAG (per 100 } \\
\text { pts) }\end{array}$ & 100 & 100 & 75 & 38 \\
$\begin{array}{l}\text { No. of patients undergoing PCI (per 100 } \\
\text { pts) }\end{array}$ & 62 & 29 & 54 & 33 \\
$\begin{array}{l}\text { Vessels treated by PCI (per 100 pts) } \\
\text { Costs per patient }\end{array}$ & 80 & 37 & 72 & 48 \\
\begin{tabular}{l}
1 year event rate \\
\hline
\end{tabular} & $2.4 \%$ & $1.9 \%$ & $2.2 \%$ & $1.9 \%$ \\
\hline
\end{tabular}


Fig. 2 Combined per-patient cost and projected 1 year event rate (death/MI) for 4 clinical pathways modeled in this study. $\mathrm{FFR}_{\text {cath }}$ and $\mathrm{FFR}_{\mathrm{CT}}$ guided clinical pathways demonstrated the lowest cost and clinical event rate compared to visualguided treatment strategies

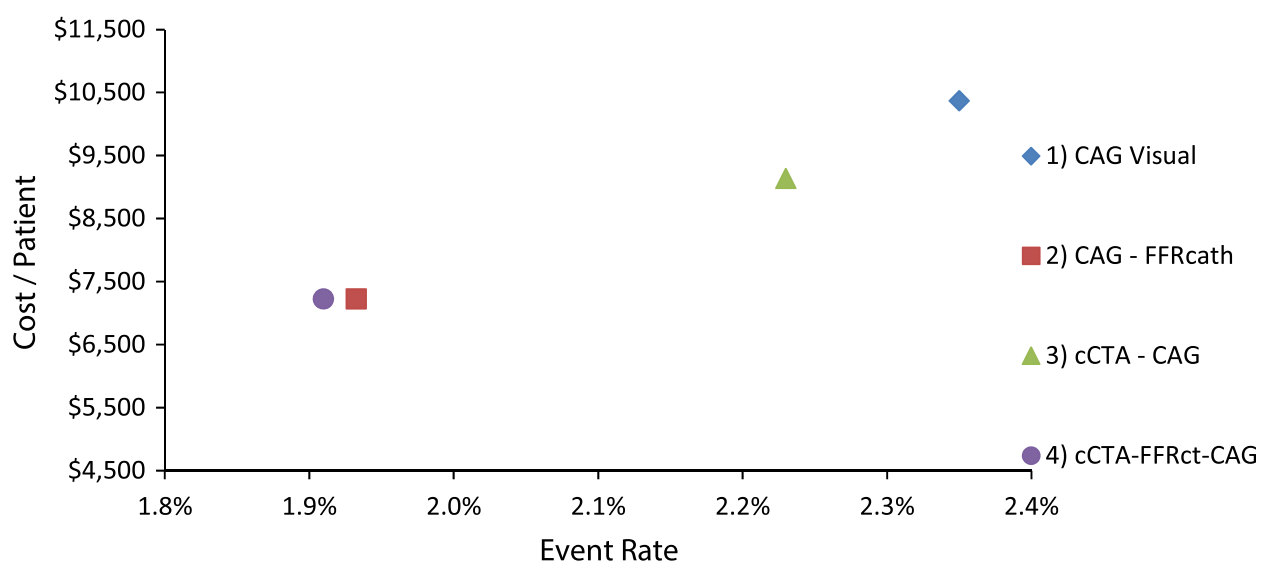

ischemia using FFR $_{\text {cath }}$ as the reference standard with significant improvement in the ability to discriminate patients with and without ischemia when compared to cCTA [5-7] and CAG [7]. The use of invasive FFR cath $_{\text {to }}$ identify lesion-specific ischemia and guide coronary intervention is now well-established and has been shown to not only improve patient outcome but also reduce costs [1]. It has been suggested that non-invasive $\mathrm{FFR}_{\mathrm{CT}}$ may be poised to assume the role of gatekeeper to the interventional catheterization laboratory, especially for intermediate stenosis [15], and a previously modeled analysis based on US data has suggested that the use of FFR $_{C T}$ may improve patient outcomes while reducing healthcare costs [8].

In this study we based our analysis on the most recently published experience with $\mathrm{FFR}_{\mathrm{CT}}$ which incorporates the latest refinements in software technology with automated image processing and improved physiologic modeling of coronary flow parameters [7]. The results show that the diagnostic performance of non-invasive $\mathrm{FFR}_{\mathrm{CT}}$ compares favorably to invasive $\mathrm{FFR}_{\text {cath }}$ and can discriminate ischemia-causing stenoses from non-functional stenoses. According to European Society of Cardiology [16] and American Heart Association [17] practice guidelines, FFR $_{\text {cath }}$ is the gold standard for assessing the hemodynamic significance of coronary lesions and for interventional clinical decision-making; however, $\mathrm{FFR}_{\text {cath }}$ is not practical in many cases for reasons of safety and time. FFR $_{\mathrm{CT}}$ has potential value in selecting patients for CAG and interventional treatment with the potential of achieving significant reduction in costs and improving outcomes compared to visual angiography-guided treatment. As indicated by the model in this study, the utilization of FFR $_{\mathrm{CT}}$ in Japan may result in fewer diagnostic catheterizations, fewer inappropriate PCI treatments, improved patient outcomes, and a $30 \%$ reduction in average cost per patient relative to standard care (Pathway 1) if fully implemented.
In addition to advancing patient care, utilizing FFR $_{\mathrm{CT}}$ technology in Japan may provide significant cost savings for the overall Japanese Healthcare System by safely deferring unnecessary CAG and identifying patients who would benefit from PCI. In 2011 it is estimated that 504,476 coronary angiographies and 181,991 non-emergent PCIs were performed in Japan [18]. If our analysis can be extrapolated to the larger population, the utilization of $\mathrm{FFR}_{\mathrm{CT}}$ might decrease coronary angiographies by as much as $\sim 60 \%$ and PCI procedures by $\sim 40 \%$. Based on the results in this study, we estimate that widespread implementation of FFR $_{C T}$ in Japan has the potential to result in considerable cost savings to the Japanese Healthcare System while improving the clinical outcome for patients.

\section{Study limitations}

This study has several important limitations. First, this study is a simulation of possible costs and outcomes rather than documentation of costs incurred and outcomes experienced utilizing $\mathrm{FFR}_{\mathrm{CT}}$ in actual clinical practice. While direct assessment of cost-efficacy is not yet available for $\mathrm{FFR}_{\mathrm{CT}}$ or other non-invasive testing modalities in stable $\mathrm{CAD}$, the source data for this study represents the largest clinical experience of any non-invasive testing modality using $\mathrm{FFR}_{\text {cath }}$ as the reference standard. FFR $\mathrm{CT}_{\mathrm{T}}$ has close direct correlation to measured FFR, which has well-documented outcome and cost data in more than 1,005 patients [11], but independent confirmation with actual outcome data utilizing $\mathrm{FFR}_{\mathrm{CT}}$ for clinical decision making is needed. The extent to which patients analyzed in this report may not precisely reflect the spectrum of patients undergoing CAG in Japan may limit the ability to extrapolate directly to expected outcomes in Japan. A prospective longitudinal study evaluating clinical outcomes, resource utilization and quality of life of $\mathrm{FFR}_{\mathrm{CT}}$-guided evaluation 
and treatment of patients with suspected CAD is currently underway (PLATFORM trial, clinical trials.gov NCT01943903). Second, this study did not include patients with acute coronary ischemia, patients with prior PCI or CABG, and patients who are not suitable candidates for cCTA. Thus, the usefulness of $\mathrm{FFR}_{\mathrm{CT}}$ in this broader population of patients with CAD is unknown. Third, costs related to clinical adverse events during follow-up were not considered; however, the $\mathrm{FFR}_{\mathrm{CT}}$ guided pathway had the lowest event rate during follow-up. Fourth, $\mathrm{FFR}_{\mathrm{CT}}$ is not yet widely available and market pricing for this test has not yet been determined. This analysis uses an $\mathrm{FFR}_{\mathrm{CT}}$ price of $\$ 2,000$, the price at which the costs of Pathways 2 and 4 were equivalent. The resulting average total cost of treating a patient in Pathway 4 (cCTA-FFR $\mathrm{CT}-\mathrm{CAG}$ ) is $\$ 7,222$. If the price of $\mathrm{FFR}_{\mathrm{CT}}$ is modeled as $\$ 1,500$, the average cost of treating a patient in this pathway decreases to $\$ 6,848$, a $34 \%$ savings compared to standard care (Pathway 1). If the price of $\mathrm{FFR}_{\mathrm{CT}}$ is modeled as $\$ 2,500$, the average cost for a patient in this pathway is $\$ 7,596$, a $27 \%$ reduction compared to standard care (Pathway 1). Finally, this analysis does not consider the possibility of only partial or limited adoption of the $\mathrm{FFR}_{\mathrm{CT}}$ decision pathway, which inherently would limit the potential cost savings of this approach. For example, the decision to send a patient to CAG is made by comprehensive evaluation of the patient's symptoms and physical findings, risk profile, and results of other non-invasive tests for myocardial ischemia and may cause the physician to override the results of the $\mathrm{FFR}_{\mathrm{CT}}$. Similarly, physicians in Pathway 2 may choose to-not measure FFR in the cath lab or may override the results of $\mathrm{FFR}_{\text {cath }}$ thus reducing the potential economic and outcome benefit. Thus, actual savings achieved may be limited by physicians' adherence to the clinical decision making pathway. Further evaluations including prospective outcome studies are underway to better understand and quantify the potential clinical and economic improvements identified in this simulation.

\section{Conclusion}

Analysis of data from the HeartFlowNXT trial and using Japanese costs of goods and services suggest that utilization of non-invasive $\mathrm{FFR}_{\mathrm{CT}}$ for clinical decision making could improve clinical outcomes and decrease costs by more accurately identifying patients for CAG and PCI.

\section{Acknowledgments The NXT trial was funded by the HeartFlow,} Inc.

Conflict of interest Ben Forrest and Christopher K. Zarins are employees of HeartFlow, Inc.

\section{References}

1. Fearon WF, Bornschein B, Tonino PA, Gothe RM, Bruyne BD, Pijls NH, et al. Fractional flow reserve versus angiography for multivessel evaluation study I. Economic evaluation of fractional flow reserve-guided percutaneous coronary intervention in patients with multivessel disease. Circulation. 2010;122:2545-50.

2. Pijls NH, Fearon WF, Tonino PA, Siebert U, Ikeno F, Bornschein $\mathrm{B}$, et al. Fractional flow reserve versus angiography for guiding percutaneous coronary intervention in patients with multivessel coronary artery disease: 2-year follow-up of the fame (fractional flow reserve versus angiography for multivessel evaluation) study. J Am Coll Cardiol. 2010;56:177-84.

3. Fearon WF, Shilane D, Pijls NH, Boothroyd DB, Tonino PA, Barbato E, et al. Fractional flow reserve versus angiography for multivessel evaluation I. Cost-effectiveness of percutaneous coronary intervention in patients with stable coronary artery disease and abnormal fractional flow reserve. Circulation. 2013;128:1335-40.

4. Kleiman NS. Bringing it all together: integration of physiology with anatomy during cardiac catheterization. J Am Coll Cardiol. 2011;58:1219-21.

5. Koo BK, Erglis A, Doh JH, Daniels DV, Jegere S, Kim HS, et al. Diagnosis of ischemia-causing coronary stenoses by noninvasive fractional flow reserve computed from coronary computed tomographic angiograms. Results from the prospective multicenter discover-flow (diagnosis of ischemia-causing stenoses obtained via noninvasive fractional flow reserve) study. J Am Coll Cardiol. 2011;58:1989-97.

6. Min JK, Leipsic J, Pencina MJ, Berman DS, Koo BK, van Mieghem C, et al. Diagnostic accuracy of fractional flow reserve from anatomic ct angiography. JAMA. 2012;308:1237-45.

7. Nørgaard BL, Leipsic J, Gaur S, Seneviratne S, Ko BS, Ito H, Jensen JM, Mauri L, De Bruyne B, Bezerra H, Osawa K, Marwan M, Naber C, Erglis A, Park SJ, Christiansen EH, Kaltoft A, Lassen JF, Bøtker HE, Achenbach S; NXT Trial Study Group. Diagnostic performance of noninvasive fractional flow reserve derived from coronary computed tomography angiography in suspected coronary artery disease: the NXT trial (Analysis of Coronary Blood Flow Using CT Angiography: Next Steps). J Am Coll Cardiol. 2014;63(12):1145-55.

8. Hlatky MA, Saxena A, Koo BK, Erglis A, Zarins CK, Min JK. Projected costs and consequences of computed tomographydetermined fractional flow reserve. Clin Cardiol. 2013;36:743-8.

9. Ministry of Health, Labour and Welfare. http://www.Mhlw.Go. Jp/bunya/iryouhoken/iryouhoken14/index.html.

10. Pijls NH, van Schaardenburgh P, Manoharan G, Boersma E, Bech JW, van't Veer M, et al. Percutaneous coronary intervention of functionally nonsignificant stenosis: 5-year follow-up of the defer study. J Am Coll Cardiol. 2007;49:2105-11.

11. Tonino PA, De Bruyne B, Pijls NH, Siebert U, Ikeno F, van' $t$ Veer M, et al. Fractional flow reserve versus angiography for guiding percutaneous coronary intervention. $\mathrm{N}$ Engl $\mathrm{J}$ Med. 2009;360:213-24.

12. Tonino PA, Fearon WF, De Bruyne B, Oldroyd KG, Leesar MA, Ver Lee PN, et al. Angiographic versus functional severity of coronary artery stenoses in the fame study fractional flow reserve versus angiography in multivessel evaluation. J Am Coll Cardiol. 2010;55:2816-21.

13. Stone GW, Maehara A, Lansky AJ, de Bruyne B, Cristea E, Mintz GS, et al. A prospective natural-history study of coronary atherosclerosis. N Engl J Med. 2011;364:226-35.

14. Pijls NH, Sels JW. Functional measurement of coronary stenosis. J Am Coll Cardiol. 2012;59:1045-57. 
15. Hecht HS. The game changer? J Am Coll Cardiol. 2014;63(12):1156-8.

16. Wijns W, Kolh P, Danchin N, Di Mario C, Falk V, Folliguet T, et al. Guidelines on myocardial revascularization. Eur Heart J. 2010;31(20):2501-55.

17. Levine GN, Bates ER, Blankenship JC, Bailey SR, Bittl JA, Cercek B, et al. American College of Cardiology Foundation; American Heart Association Task Force on Practice Guidelines;
Society for Cardiovascular Angiography and Interventions. J Am Coll Cardiol. 2011;58(24):e44-122.

18. JCS National Survey on Management of Cardiovascular Diseases: Annual Report. http://www.J-circ.Or.Jp/jittai_chosa/jittai_ chosa2011web.Pdf (2011). 